\section{Turn-over orbital septal flap and levator recession for upper-eyelid retraction secondary to thyroid eye disease}

${ }^{1}$ Department of Ophthalmology، Kyoto Prefectural University of Medicine, Kyoto, Japan

${ }^{2}$ Department of Ophthalmology and Visual Sciences, South Australian Institute of Ophthalmology, Adelaide University, Adelaide, South Australia, Australia

\section{${ }^{3}$ Department of Oculoplastic} and Orbital Surgery, Seirei Hamamatsu Hospital,

Hamamatsu, Japan

Correspondence:

PN Shams, South Australian Ophthalmology Network, Royal Adelaide Hospital, Level 8, East Wing, Adelaide, South Australia 5000, Australia Tel: + 6188222 2729; Fax: + 6188222 2741;

E-mail: pari.shams@gmail.com

This study has been presented at the Royal Australian and New Zealand College of Ophthalmologists annual meeting, November 2012, Melbourne, Australia and at the British Oculoplastic Surgeons Society Meeting, June 2013. Bristol, United Kingdom.

Received: 22 November 2012 Accepted in revised form: 2 July 2013

Published online: 2 August 2013

\begin{abstract}
Background A turn-over septal flap has been reported as a spacer for levator lengthening in a single case report. This study reports the preliminary outcomes of this technique in a series of patients with upper-lid retraction (ULR) associated with thyroid eye disease (TED) causing symptomatic exposure keratopathy (EK).

Methods Retrospective, multicenter study of 12 eyelids of 10 patients with TED undergoing a transcutaneous levatorlengthening technique using the reflected orbital septum (OS) as a spacer. Change in palpebral aperture (PA) and contour, position of the skin crease (SC), symptoms of EK, and complications were recorded.

Results The average age was 47.5 years. Two patients were excluded, as their septa were found to be very thin at surgery. At an average of 13 months postoperatively, the PA was reduced by $2.5 \mathrm{~mm}$ on average $(P<0.001)$ and was within $1 \mathrm{~mm}$ of the contralateral eyelid in 11 cases $(92 \%)$; the position of the SC was within $1 \mathrm{~mm}$ of the desired position in all cases. EK resolved in all cases. Complications included one case of overcorrection and one case of recurrent lateral flare.

Conclusions The turn-over orbital septal flap technique may be a viable option as an autogenous spacer for the treatment of ULR in TED. This technique may be possible in cases where the OS has been opened by previous surgery but may not be feasible in patients in whom the septum is very thin.

Eye (2013) 27, 1174-1179; doi:10.1038/eye.2013.160;

published online 2 August 2013
\end{abstract}

A Watanabe ${ }^{1}$, PN Shams ${ }^{2}$, N Katori ${ }^{3}$, S Kinoshita ${ }^{1}$ and D Selva ${ }^{2}$

Keywords: upper-eyelid retraction; orbital septal flap; levator recession

Introduction

Achieving a predictable eyelid height and contour in the surgical correction of uppereyelid retraction remains a challenge for surgeons, as evidenced by the variety of procedures reported. ${ }^{1,2}$ These techniques are based on weakening or lengthening the upper-eyelid retractors and include anterior or posterior approaches to graded recession or resection of Müller's muscle, ${ }^{3-5}$ levator aponeurosis (LA)/muscle, ${ }^{6-8}$ or both, ${ }^{5,9-14}$ full-thickness blepharotomy, ${ }^{15-17}$ levator lengthening by marginal myotomy, ${ }^{18,19}$ z-myotomy, ${ }^{20}$ castellated levator aponeurotomy, ${ }^{21}$ reattachment of the recessed levator to the tarsus by various spacers (sclera, 22,23 mersilene mesh, ${ }^{24,25}$ sutures, ${ }^{26,27}$ adjustable sutures, ${ }^{28,29}$ orbital septal flap, ${ }^{30}$ pretarsal soft tissues, ${ }^{31}$ and deep temporal fascia ${ }^{32}$ ), pedicle tarsal rotation flap, ${ }^{33}$ or medial transposition of the lateral horn of the LA. ${ }^{34}$

The orbital septum (OS) arises from the arcus marginalis and terminates inferiorly as it attaches to the LA; it can be used as a vascularized turn-over flap to act as a spacer between the recessed LA-Muller's muscle complex-Muller's muscle complex and the tarsal plate. This technique was first described by Lai et $a l^{30}$ in a case report. In this study, we describe the preliminary outcomes from the use of this technique in a series of patients and 
assess the feasibility of creating a flap in a variety of cases with upper-eyelid retraction.

\section{Materials and methods}

This retrospective, multicenter study was conducted at the Department of Ophthalmology, Kyoto Prefectural University of Medicine, Kyoto, Japan, from January 2007 to January 2012 and at The Royal Adelaide Hospital, Adelaide, South Australia, Australia, from January 2009 to January 2012. At each center, surgeries were performed by a single surgeon (AW in Kyoto and DS in Adelaide).

Inclusion criteria included consecutive patients requiring surgical correction of upper-eyelid retraction secondary to thyroid eye disease (TED) and no previous upper-eyelid surgery. Written informed consent was obtained from all patients. Cases in which the OS was found to be thin or intraoperatively dehiscent, and was considered by the surgeon to be unsuitable for a flap, were excluded. The patient records and images were examined and the patient's age, gender, thyroid status, and total length of postoperative follow-up recorded.

Clinical symptoms and signs of exposure keratopathy (EK) (lagophthalmos, foreign body sensation, and photophobia) and poor cosmesis were recorded as present or absent. The palpebral aperture (PA), upper marginal reflex distance (MRD) (the distance from the mid-pupil to the upper eyelid at the 12 o'clock position), distance of the skin crease (SC) from the center of the eyelid margin, and upper and lower scleral show were recorded for the operated eyelid. The presence or absence of a smooth upper eyelid margin contour and lateral flare was noted. The presence of corneal epitheliopathy was graded as mild, moderate, or severe. The differences in PA, lid margin contour, and SC symmetry between the operated and contralateral eyelid were recorded preoperatively and at the final follow-up visit. Patientreported satisfaction with the surgical result was recorded as 'yes' or 'no', and the postoperative eyelid position was graded according to Mourits and Sasim. ${ }^{35}$ Complications associated with the surgery were recorded. Statistical analyses were performed using Sigmaplot version 12.5 for Windows (Systat Software Inc., San Jose, CA, USA). Written informed consent was obtained from all patients. Institutional review board ethics approval was obtained for the study. The research was conducted in adherence to the tenets of the Declaration of Helsinki.

\section{Surgical technique}

The upper-eyelid SC is marked to achieve symmetry with the contralateral side, and the skin is injected with 0.5\% bupivocaine with 1:200 000 adrenaline. A 3-cm skin incision is made along the marked SC and the orbicularis muscle is divided to expose the tarsal plate (Figure 1a). The OS and LA are identified. The LA-Muller's complex is hydrodissected from the underlying conjunctiva with normal saline. Using blunt-tipped Westcott scissors, Müller's muscle is dissected from the underlying conjunctiva in a blunt upward stripping motion with the blades of the scissors slightly apart, from just above the superior tarsal arcade to its superior origin from the levator. The LA-Muller's complex is then detached from the tarsus at a level immediately above the upper border of the tarsal plate without opening the OS (Figure 1b); a hot wire cautery can be used instead of the Westcott
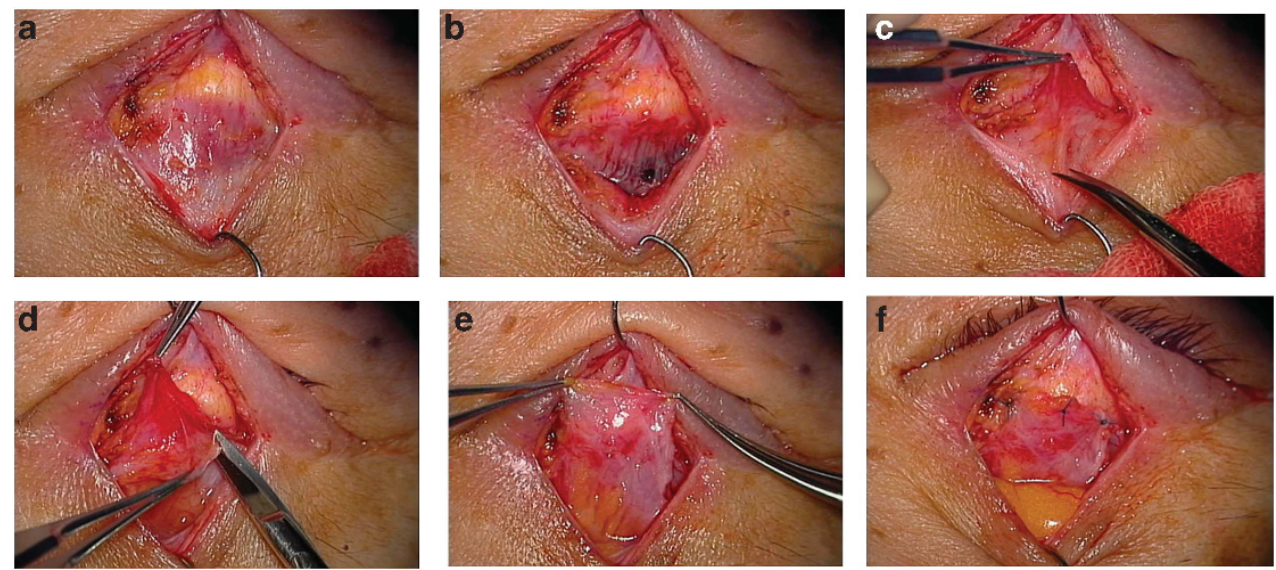

Figure 1 (a) An upper lid skin crease incision is made and the orbicularis muscle is divided to expose the tarsal plate. (b) The levator aponeurosis-Muller's complex has been dissected off the conjunctiva and detached from the upper border of the tarsal plate. (c) The septum is opened at the arcus marginalis $\sim 15 \mathrm{~mm}$ above the levator aponeurosis. (d) The medial and lateral horns of the orbital septum are identified and divided. (e) The superior border the orbital septum is reflected inferiorly. (f) The distal end of the orbital septal flap is then anchored to the superior border of the tarsal plate. 
scissors for improved hemostasis. The medial and lateral horns of the LA are also identified and divided. The OS lies beneath the orbicularis muscle. It is ballooned away from the muscle with a small amount of local anesthetic solution to facilitate dissection. Upward traction on the orbicularis muscle facilitates dissection of the septum. The OS is detached from the arcus marginalis $\sim 15 \mathrm{~mm}$ above the white line of the LA (Figure 1c), its medial and lateral borders are incised (Figure 1d), and it is then reflected inferiorly (Figure 1e). The septal flap is then sutured to the superior one-third of the tarsal plate using adjustable $6 / 0$ vicryl sutures (Figure 1f); two or three sutures are used to achieve the correct contour. The length of the OS spacer is titrated according to the intraoperative lid height in the same manner as the levator advancement surgery. The distal edge of the septal flap is then included in the orbicularis closure in an attempt to create a dynamic $\mathrm{SC}$ at the level of the skin incision.

Alternatively, a flap can be combined with a graded recession in the context of anterior blepharotomy. Hence, following the creation of a septal flap, the LA can be released at the tarsus and dissected off Müller's muscle. A mullerectomy is then performed, and a graded recession of the levator can be performed as dictated by titration against the intraoperative lid height. The conjunctiva can be divided as per a graded full-thickness blepharotomy for more severe cases of retraction, although this was not performed on any case in this series. The septal flap can then be sutured to the tarsus, with sutures being adjusted or added as necessary for adequate contour and to avoid a flattened contour. Figure 2 shows the pre- and post-operative clinical photographs of an Asian patient with TED.

\section{Results}

Following the exclusion of two patients, aged 70 and 81 years, from the study, in whom the OS was found to be too thin to be used as a flap, 12 of 10 patients (two bilateral and eight unilateral cases) underwent levator recession using the described turn-over orbital septal flap technique in which the septum was of adequate thickness. Four patients were Caucasian and the other six were Asian. The average age was $47.5 \pm 13$ years (range 24-76) and eight patients (67\%) were female. No cases had undergone previous upper-eyelid surgery. Seventy percent of patients $(n=7 / 10)$ were euthyroid at the time of surgery and $70 \%$ had received steroid treatment for that condition. The average follow-up was 13 months (range 6-20). The indication for eyelid lowering was lagophthalmos in five (42\%) (mean degree of lagophthalmos was $1.9 \mathrm{~mm}$ (range 1-6.5 mm)), foreign body sensation in eight $(67 \%)$, photophobia in seven $(58 \%)$, and symptomatic epiphora in three (25\%). All patients were significantly troubled by their cosmetic appearance. Table 1 shows the pre- and postoperative eyelid measurements and the mean change in PA, upper MRD, upper and lower scleral show, and SC at a mean follow-up of 13 months postoperatively. There was a statistically significant reduction in the mean PA of $2.5 \pm 1.0 \mathrm{~mm}(P<0.001)$ and a reduction in mean upper MRD of $2.5 \pm 1.4 \mathrm{~mm}(P<0.0001)$. The intended upper MRD was determined by the MRD in the contralateral eyelid, which in 10 cases (83\%) was un-operated. The upper scleral show was reduced by an average of $1.7 \mathrm{~mm}$ $(P<0.001)$ and there was no change in the lower scleral show as would be expected. The average height of the SC remained within $1 \mathrm{~mm}$ of the intended position set at surgery in all cases at a mean follow-up of 13 months postoperatively ( $-0.1 \mathrm{~mm}$ change, $P=0.61$ ), and in 10 cases $(83 \%)$ it remained unchanged. Preoperatively, the mean difference in PA between the two eyes was $1.4 \mathrm{~mm}$ $(0-3 \mathrm{~mm})$, and this was reduced by a mean of $1.2 \mathrm{~mm}$ down to $0.2 \mathrm{~mm}$ postoperatively $(P<0.003)$. Figure 3 is a scatter plot of the pre- and postoperative PA. The PA was within $1 \mathrm{~mm}$ of the contralateral eyelid in 11 cases (92\%). Postoperative symmetry in SC and eyelid margin contour with the contralateral eyelid was present in $10(83 \%)$ and 11 eyelids (92\%), respectively.

Five cases $(42 \%)$ had an average postoperative lagophthalmos of $0.4 \mathrm{~mm}$, which spontaneously resolved


Figure 2 (a) Preoperative clinical photograph of a 54-year-old patient with right-upper-eyelid retraction due to thyroid eye disease. (b) Postoperative clinical photograph of the patient following a turn-over orbital septal flap. 
Table 1 Summary of postoperative change in eyelid parameters

\begin{tabular}{lcc}
\hline Eyelid parameters & $\begin{array}{c}\text { Mean change } \\
(\mathrm{mm})\end{array}$ & P-value \\
\hline Palpebral aperture & -2.5 & $<0.001$ \\
Upper marginal reflex distance & -2.5 & $<0.001$ \\
Upper scleral show & -1.7 & $<0.001$ \\
Lower scleral show & 0 & $=1$ \\
Skin crease & -0.1 & $=0.6$ \\
$\begin{array}{l}\text { Difference in palpebral aperture } \\
\text { between eyelids }\end{array}$ & -1.6 & $=0.003$ \\
\end{tabular}

\section{Scatter plot showing pre and post-operative palpebral aperture}

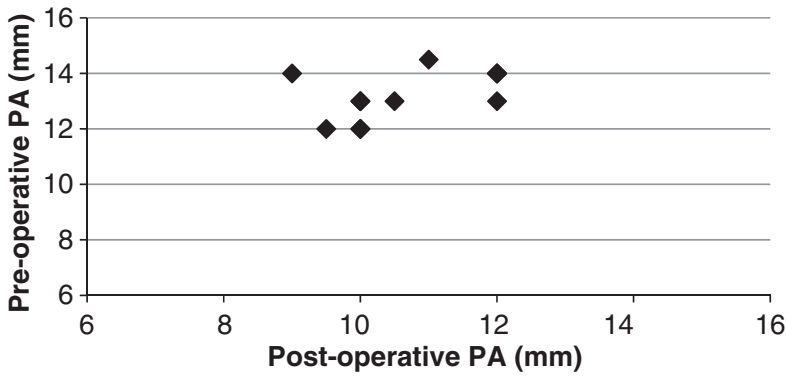

Figure 3 Scatter plot of the preoperative PA and post-turn-over orbital septal flap and levator recession for upper-eyelid retraction secondary to thyroid eye disease.

by 1-2 weeks postoperatively. All patients experienced a resolution of epiphora and photophobia. There was a significant improvement in signs of corneal exposure; preoperatively, there was evidence of mild corneal punctate epitheliopathy in three cases $(25 \%)$, moderate corneal punctate epitheliopathy in one case $(8 \%)$, and severe in one case $(8 \%)$; seven cases $(58 \%)$ had no keratopathy.

Postoperatively, only two cases (17\%) had mild epitheliopathy and 10 cases $(83 \%)$ had no evidence of keratopathy $(P=0.01)$.

The postoperative eyelid position was graded according to Mourits and Sasim ${ }^{35}$ and found to be 'perfect' in seven (70\%) patients, 'acceptable' in two (20\%) and a 'failure' in one (10\%); this was because of overcorrection in a patient who underwent a second procedure (septal flap and levator were identified and advanced to correct the overcorrection), achieving a good result at 4 months post procedure. Another patient who initially had an acceptable result developed late recurrence of lateral flare, but has undergone no further surgery. There were no other significant complications from this procedure. At the latest follow-up visit, nine patients (90\%) were satisfied with the surgical outcome.

\section{Discussion}

In this preliminary study, we have reported the results of surgery using a turn-over orbital septal flap combined with levator recession in patients with upper-eyelid retraction due to TED.

The results of eyelid lowering using an orbital septal flap in this study compares favorably with those of other well-established techniques. Using the graded fullthickness anterior blepharotomy procedure, first communicated by Koornneef in 1999, Elner et al ${ }^{15}$ reported an average postoperative MRD of $3.07-3.57 \mathrm{~mm}$ and $93 \%$ patient satisfaction. A series by Hintschich and Haritoglou ${ }^{16}$ reported that $95 \%$ of operated lids achieved a 'perfect' or an 'acceptable' result while using full-thickness blepharotomy to treat TED lid retraction, which compares well to the results reported with this technique. Mourits and Sasim ${ }^{35}$ described a modified Harvey's technique ${ }^{10}$ by dissecting the aponeurosis together with Müller's muscle off the tarsus and the conjunctiva from lateral to medial only to the extent necessary to achieve an acceptable position and contour of the eyelid in the upright position. They reported a perfect result in 50\%, an acceptable result in $32 \%$, and an unacceptable result in $18 \%$ of patients. Looi et al's $^{14}$ report on posterior approach graded recession or resection of Müller's muscle as well as LA and recorded a mean pre- and postoperative upper MRD of 7.3 and $4.3 \mathrm{~mm}$, respectively, with $89 \%$ achieving the desired result of an upper MRD of $4 \pm 1 \mathrm{~mm}$ after one procedure. The average postoperative asymmetry was $0.2 \mathrm{~mm}$, which compares well with this series $(0.2 \mathrm{~mm})$. Some techniques of levator recession are associated with recession of SC postoperatively. Our experience with the turn-over orbital septal flap has been of good control and stability of SC position, with 10 cases (83\%) having an unaltered SC position at final follow-up and all cases being within $1 \mathrm{~mm}$ of the intended SC position. Moreover, in 11 eyelids (92\%) there was good symmetry of eyelid margin contour with the contralateral eyelid.

The use of an orbital septal flap as a spacer to lengthen the LA may not be possible in all patients; the OS is a fibrous, multilaminar sheet of variable strength, reinforced by the ligaments beneath its posterior surface and the submuscular fibroadipose tissue in front. ${ }^{36}$ It is of variable thickness, being relatively thick in the young and quite easily identified at surgery, but may be attenuated and dehiscent in older patients. ${ }^{37}$ This was the case in two older Caucasians with TED in this series. Whether TED also alters the properties of the OS as a suitable spacer requires further investigation.

The surgeon must therefore be prepared to proceed with another technique to lower the eyelid should the septum appear thin and attenuated at the time of surgery.

Anatomical variations may also exist in the thickness of the OS between races. Although it has been the authors' impression that Asian eyelids may display a thicker septum, this has not been specifically reported in the literature to the best of our knowledge. It is the authors' 
experience that the OS can, on occasion, be successfully utilized as a flap even in patients who have undergone previous anterior-approach ptosis surgery. A scarred membrane is often visible over the anterior LA, which can be equally utilized as a spacer; therefore, the quality of the septum should be assessed at the time of surgery.

The use of a septal flap may also be especially useful in cases following ptosis overcorrection in which a large levator resection has been performed necessitating the use of a spacer or hang-back sutures. In milder cases of eyelid retraction, a spacer is perhaps of less value. For more severe cases of retraction where a large recession is required, spacers may possibly decrease the chance of recurrent retraction, which occurs because of scarring between the recessed end of the retractors and the tarsus. A spacer also provides a means of connection between the tarsus and retractors, which may help with the control of the eyelid contour. A further putative advantage may be that the flap enables communication of the levator action to the desired SC, which may enhance the ability to control the SC height in eyelid retraction surgery. However, in the absence of randomized controlled trials for any surgical techniques for eyelid retraction, all the postulated advantages of the use of a spacer over alternate techniques remain speculative.

To successfully identify the OS, it helps to understand where the OS joins or fuses with the LA; this varies from a position $\sim 3-5 \mathrm{~mm}$ above the superior aspect of the tarsal plate to as far as $10-15 \mathrm{~mm}$ above the tarsal plate, ${ }^{38}$ and is higher in Europeans than in Asians. ${ }^{39}$ Therefore, after making the eyelid SC incision, one must dissect $\sim 5 \mathrm{~mm}$ superiorly in order to consistently identify the OS and LA before they join. Caution must be exercised in patients who have undergone previous upper-eyelid surgery.

The desired exact width of the orbital septal flap may not be of critical importance but should be centered in line with the pupil and should be of sufficient width to confer a good eyelid contour. The length of the flap is determined by the degree of eyelid retraction; a report by Lai $e a^{30}$ suggested the length of the flap be calculated as $2 \times$ (degree of retraction in $\mathrm{mm}$ ) $+2 \mathrm{~mm}$, a modification from the report by Piggot et al. ${ }^{21}$ For example, to correct a 2-mm retraction, the length of the flap would be $6 \mathrm{~mm}$. This formula was not used in any cases in this series but can be a useful guide. If the available height of the septum is insufficient, one could consider the option of tenotomy of either the LA or the septal flap in the same manner as described for levator myotomy. ${ }^{18}$

We have found the orbital septal flap to be a useful spacer for interposing between the recessed levator complex and the tarsal plate for several reasons; the anterior approach to the eyelid using this technique will be familiar to surgeons, providing good surgical access and visualization, making the dissection of the orbital septal flap relatively straightforward. The OS is readily available in the majority of patients and can be utilized in an atraumatic manner and from the same surgical site without additional donor site morbidity associated with harvesting a graft. The length of the septal flap can be varied to modulate the extent of desired correction. The OS has the advantage of being vascularized, and therefore is a viable structure that appears not to undergo resorption, unlike the sclera, which can result in unpredictable outcomes and a thickened appearance. Furthermore, compared with other spacer materials such as a mersilene mesh, ${ }^{25}$ it does not carry the risk of extrusion and possibly has a higher success rate $(80 \%$ versus $90 \%$ in this study). The reattachment of the LA via the turn-over septal flap into its normal anatomical insertion site may help maintain eyelid contour and enable a graded adjustment of the eyelid height. The turn-over orbital septal flap technique also appears to have the advantage of permitting control over the SC.

The turn-over septal flap provides a readily available alternative to hang-back sutures or other non-autogenous spacers for eyelid-retraction surgery. The preliminary outcomes of this technique in patients with TED appear to be acceptable; however, further experience will be necessary to ascertain whether it carries any significant advantage over alternate spacers in terms of predictability and contour, and which group of patients is most likely to benefit from this technique.

\section{Summary}

\section{What was known before}

- Achieving a predictable eyelid height and contour in the treatment of upper-eyelid retraction in TED remains a challenge.

- Techniques are based on weakening or lengthening the upper-eyelid retractors with or without reattachment of the recessed levator to the tarsus by various spacers.

- The use of the orbital septum as a vascularized turn-over flap to act as a spacer has been described in a single case report.

\section{What this study adds}

- The turn-over septal flap is a readily available alternative to hang-back sutures or other non-autogenous spacers for eyelid retraction surgery in TED. It enables a graded adjustment of eyelid height, may help maintain eyelid contour, and permit better control over the skin crease.

- The use of an orbital septal flap as a spacer may not be possible in all patients.

- Further experience will need to ascertain whether it carries any advantage over alternate spacers in terms of predictability and contour, and which group of patients is most likely to benefit from this technique.

\section{Conflict of interest}

The authors declare no conflict of interest. 


\section{References}

1 Cruz AA, Ribeiro SF, Garcia DM, Akaishi PM, Pinto CT. Graves upper eyelid retraction. Surv Ophthalmol 2013; 58(1): 63-76.

2 Kazim M, Gold KG. A review of surgical techniques to correct upper eyelid retraction associated with thyroid eye disease. Curr Opin Ophthalmol 2011; 22(5): 391-393.

3 Henderson JW. Relief of eyelid retraction: a surgical procedure. Arch Ophthalmol 1965; 74(2): 205-216.

4 Putterman AM, Urist M. Surgical treatment of upper eyelid retraction. Arch Ophthalmol 1972; 87(4): 401-405.

5 Putterman AM. Surgical treatment of thyroid-related upper eyelid retraction. Graded Muller's muscle excision and levator recession. Ophthalmology 1981; 88(6): 507-512.

6 Callahan A. Levator recession: with reattachment to the tarsus with collagen film. Arch Ophthalmol 1965; 73(6): 800-802.

7 Putterman AM, Urist MJ. A simplified levator palpebrae superioris muscle recession to treat overcorrected blepharoptosis. Am J Ophthalmol 1974; 77(3): 358-366.

8 Baylis HI, Cies WA, Kamin DF. Correction of upper eyelid retraction. Am J Ophthalmol 1976; 82(5): 790-794.

9 Moran RE. The correction of exophthalmos and levator spasm. Plast Reconstr Surg (1946) 1956; 18(6): 411-426.

10 Harvey JT, Corin S, Nixon D, Veloudios A. Modified levator aponeurosis recession for upper eyelid retraction in Graves disease. Ophthalmic Surg 1991; 22(6): 313-317.

11 Levine MR, Chu A. Surgical treatment of thyroid-related lid retraction: a new variation. Ophthalmic Surg 1991; 22(2): 90-94.

12 Older JJ. Surgical treatment of eyelid retraction associated with thyroid eye disease. Ophthalmic Surg 1991; 22(6): 318-322; (discussion 322-3).

13 Liu D. Surgical correction of upper eyelid retraction. Ophthalmic Surg 1993; 24(5): 323-327.

14 Looi AL, Sharma B, Dolman PJ. A modified posterior approach for upper eyelid retraction. Ophthal Plast Reconstr Surg 2006; 22(6): 434-437.

15 Elner VM, Hassan AS, Frueh BR. Graded full-thickness anterior blepharotomy for upper eyelid retraction. Trans Am Ophthalmol Soc 2003; 101: 67-73; (discussion 73-5).

16 Hintschich C, Haritoglou C. Full thickness eyelid transsection (blepharotomy) for upper eyelid lengthening in lid retraction associated with Graves' disease. Br J Ophthalmol 2005; 89(4): 413-416.

17 Shortt AJ, Bhogal M, Rose GE, Shah-Desai S. Stability of eyelid height after graded anterior-approach lid lowering for dysthyroid upper lid retraction. Orbit 2011; 30(6): 280-288.

18 Grove Jr AS. Eyelid retraction treated by levator marginal myotomy. Ophthalmology 1980; 87(10): 1013-1018.

19 Grove Jr AS. Levator lengthening by marginal myotomy. Arch Ophthalmol 1980; 98(8): 1433-1438.
20 Collin JR, Allen L, Castronuovo S. Congenital eyelid retraction. Br J Ophthalmol 1990; 74(9): 542-544.

21 Piggot TA, Niazi ZB, Hodgkinson PD. New technique of levator lengthening for the retracted upper eyelid. $\mathrm{Br}$ J Plast Surg 1995; 48(3): 127-131.

22 Crawford JS, Easterbrook M. The use of bank sclera to correct lid retraction. Can J Ophthalmol 1976; 11(4): 309-322.

23 Mourits MP, Koornneef L. Lid lengthening by sclera interposition for eyelid retraction in Graves' ophthalmopathy. Br J Ophthalmol 1991; 75(6): 344-347.

24 Downes RN, Jordan K. The surgical management of dysthyroid related eyelid retraction using Mersilene mesh. Eye 1989; 3(4): 385-390.

25 Fenton S, Kemp EG. A review of the outcome of upper lid lowering for eyelid retraction and complications of spacers at a single unit over five years. Orbit 2002; 21(4): 289-294.

26 Goldstein I. Recession of levator muscle for lagophthalmos in exophthalmic goiter. Arch Ophthalmol 1934; 11: 389-393.

27 Blaskovics L. Atlas of Ophthalmic Surgery. J. B. Lippincott Co: Philadelphia, 1961; 76.

28 Hylkema HA, Koornneef L. Treatment of ptosis by levator resection with adjustable sutures via the anterior approach. Br J Ophthalmol 1989; 73(6): 416-418.

29 Collin JR, O'Donnell BA. Adjustable sutures in eyelid surgery for ptosis and lid retraction. Br J Ophthalmol 1994; 78(3): 167-174.

30 Lai CS, Lin TM, Tsai CC, Lin SD. A new technique for levator lengthening to treat upper eyelid retraction: the orbital septal flap. Aesthetic Plast Surg 2002; 26(1): 31-34.

31 Cho IC, Kang JH, Kim KK. Correcting upper eyelid retraction by means of pretarsal levator lengthening for complications following ptosis surgery. Plast Reconstr Surg 2012; 130(1): 73-81.

32 Schwarz GS, Spinelli HM. Correction of upper eyelid retraction using deep temporal fascia spacer grafts. Plast Reconstr Surg 2008; 122(3): 765-774.

33 Kohn R. Treatment of eyelid retraction with two pedicle tarsal rotation flaps. Am J Ophthalmol 1983; 95(4): 539-544.

34 Ceisler EJ, Bilyk JR, Rubin PA, Burks WR, Shore JW. Results of Mullerotomy and levator aponeurosis transposition for the correction of upper eyelid retraction in Graves disease. Ophthalmology 1995; 102(3): 483-492.

35 Mourits MP, Sasim IV. A single technique to correct various degrees of upper lid retraction in patients with Graves' orbitopathy. Br J Ophthalmol 1999; 83(1): 81-84.

36 Kakizaki H, Malhotra R, Selva D. Upper eyelid anatomy: an update. Ann Plast Surg 2009; 63(3): 336-343.

37 Zide BM, Jelks GW. Surgical anatomy of the orbit. Plast Reconstr Surg 1984; 74(2): 301-305.

38 Meyer DR, Linberg JV, Wobig JL, McCormick SA. Anatomy of the orbital septum and associated eyelid connective tissues. Implications for ptosis surgery. Ophthal Plast Reconstr Surg 1991; 7(2): 104-113.

39 Doxanas MT, Anderson RL. Oriental eyelids. An anatomic study. Arch Ophthalmol 1984; 102(8): 1232-1235. 